\title{
Cluster Load Balancing Algorithm based on Intelligent Genetic Algorithm of Vector
}

\author{
Huang Weihua ${ }^{1}$, Ma Zhong ${ }^{1}$, Dai Xinfa ${ }^{1},{\text { Gao } \mathrm{Yi}^{1} \text { and Xu Mingdi }}^{1}$ \\ ${ }^{1}$ Wuhan Digital Engineering Institute, Wuhan, 430205, China \\ 1903908425@qq.com
}

\begin{abstract}
To make load of server cluster more balanced, aimed at defect of "early-mature" existing in particle swarm optimization algorithm, a server cluster load balancing method is put forward to improve cultural particle swarm optimization algorithm. Firstly, make use of main cluster space of particle swarm optimization algorithm and knowledge space of cultural algorithm to form mechanism of "double evolution and double promotion", and to improve global searching capacity of algorithm and operational efficiency. Then bring in genetic algorithm evolution mechanism to improve evolution operation of knowledge space. Finally, the algorithm will be applied to server cluster load balancing problem to find solution. Simulation result shows that improved cultural particle swarm optimization algorithm increases resource utilization rate of server cluster system, and makes load more balanced, being an effective and reliable cluster system load balancing algorithm. At the same time, research regarding to how to embody effect of relevant parameter setting of improved cultural particle swarm optimization algorithm on solution result is problem and direction of further research.
\end{abstract}

Keywords: Load balancing; Cluster; Early-mature; Genetic algorithm; Server

\section{Introduction}

Server cluster load balancing is that equitable distribution is performed to all loads under the condition where certain constraint condition is followed, making cluster system resources fully used. Realize the purpose that total time limit of project is minimized by arranging construction starting time of all tasks of project, and it is a typical NP-hard problem[1]. Server cluster load balancing is always concerned by scholars, and many scholars perform a great deal of research to it and gain many achievements. Load balancing algorithm is mainly divided into static and dynamic balance[2]. Because static load balancing algorithm cannot correctly reflect node load change rule of server cluster system, applied range is restricted[3]. Dynamic load balancing algorithm can describe dynamic change trend of node load, becoming main balancing algorithm of present server cluster load balancing. Traditional balancing algorithms mainly are: the fastest connection algorithm and the minimum weighting connection algorithm. As for small server cluster system, traditional algorithm has relatively good performance, but as for large server cluster system, it has low efficiency, and it is easy for imbalance phenomenon[4,5] to appear in load. Aimed at NP-hard feature of server cluster load balancing, some scholars put forward server cluster load balancing methods on the basis of genetic algorithm, simulated annealing algorithm, ant colony algorithm and particle swarm optimization algorithm. They have advantages of strong swarm intelligence and search capability, which effectively improves resource utilization rate of system with relatively good load balancing[6-8]. In these algorithms, particle swarm optimization algorithm is a swarm intelligence algorithm that is successfully applied to server cluster load balancing problem[9]. However, because of defect of particle swarm optimization algorithm, it is easy to fall into local optimization and phenomenon of "early-mature" will appear, thus 
making certain difference between optimization results and ideal results exist[10]. Cultural algorithm is calculation model based on multiple evolutionary process of population, and cultural particle swarm optimization algorithm (CPSO) is a new swarm intelligence algorithm that integrates cultural algorithm to particle swarm optimization algorithm frame, achieving good effect in constraint optimization problem solution[11,12]. To solve defect of particle swarm optimization algorithm that exists in server cluster load balancing problem solution, server cluster load balancing strategy of improved cultural particle swarm optimization algorithm is put forward. Improve effectiveness and feasibility of cultural particle swarm optimization algorithm in server cluster load balancing problem solution through simulation test and verification.

\section{Load Balancing Problem}

On the basis of analysis on joint processing capacity of system, server cluster load balancing performs relatively reasonable distribution to corresponding nodes to process according to user request, to make resource of cluster system fully used, quickening completion time of user request.

Supposed that server cluster system consists of $n$ sets of servers, and they are defined as: $\{S 1, S 2, \ldots, S n\}$, and within a period of time, there are $m$ concurrent requests, then load index definition of the $i$ th set of server is as follows:

$$
\begin{aligned}
\operatorname{Load}\left(N_{i}\right)= & R_{1} * \operatorname{Lcpu}\left(N_{i}\right)+R_{2} * \operatorname{Lmemory}\left(N_{i}\right) \\
& +R_{3} * \operatorname{Lio}\left(N_{i}\right)+R_{4} * \operatorname{Lqtime}\left(N_{i}\right)
\end{aligned}
$$

In the formula, weighted value $\sum R_{i}=1$.

Supposed that $X i$ is time needed when some server processes $S i$ user requests, the optimum balancing scheme of server cluster system load is to find out the minimum value of $\sum X_{i}$, i.e.:

$T=\operatorname{Min}\left\{\sum X_{i}\right\}, 0<i \leq n$

In the formula, $X_{i}=\sum T_{j}^{i} *\left(w_{i}+R_{4} *\left(1-1 / \sum T_{j}^{i}\right)\right)$.

\section{Improved Cultural Particle Swarm Optimization Algorithm}

Cultural particle swarm optimization (CPSO) algorithm is an intelligent algorithm that integrates cultural algorithm and particle swarm optimization algorithm, and in its evolution process, particle tails after 2 goals, i.e., $p_{g}$ (overall extreme value) and $p_{\mathrm{i}}$ (present extreme value of individual) and globally optimal solution track will be formed. These optimal solutions will be considered as global knowledge information of iterative search, and knowledge space will be continuously kept and be renewed, and knowledge space will be evolved. At the same time, achieve greater global searching capacity of algorithm through double evolution and mutual effect of PSO space and knowledge space.

Because of ego evolution mechanism restriction of knowledge space in cultural algorithm, knowledge space cluster does not have ideal effect in ego evolution, and effective effect on under-layer main cluster space decreases obviously. To avoid above phenomenon, an improved cultural particle swarm optimization algorithm (ICPSO) is put forward. Apply population evolution mechanism of genetic algorithm to ego evolution of knowledge space, and perform ego evolution and update to knowledge space cluster through selection, crossover and mutation operation to improve global searching capacity and operational efficiency of ego evolution of knowledge space cluster. Frame of ICPSO algorithm is as shown in Figure 1. 


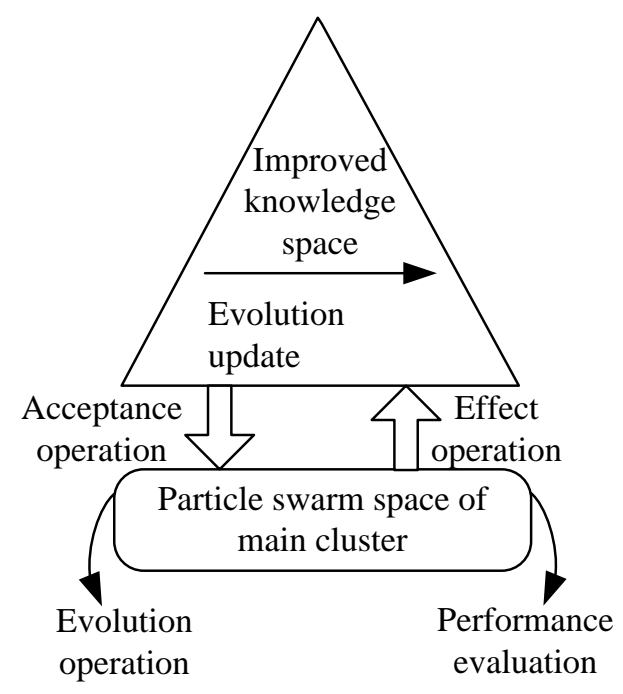

Figure 1. Frame of ICPSO Algorithm

Improve ego evolution and update of knowledge space cluster through population evolution mechanism of genetic algorithm. Adopt selection, crossover and mutation operation to perform ego evolution.

(1) Selection operation.

Step1: Calculate fitness value of each individual: $f(i), i=1,2, \ldots, n$.

Step2: Confirmation of individual selection probability $P(C i)$. Individual after order is marked as: $\{C 1, C 2, \ldots, C n\}$, and then individual selection probability is:

$$
P\left(C_{i}\right)=\frac{1}{n}\left\{\sigma^{g}-\frac{\sigma^{a}-\sigma^{b}}{n-1}(i-1)\right\}
$$

In the formula, $i$ is order No. of individual; $\sigma^{g}$ is expected number of optimal individual $C 1$ after selection operation. $\sigma^{g}=n \times P\left(C_{1}\right) \cdot \sigma^{b}$ is expected number of the worst individual $C_{n}$ after selection operation. $\sigma^{b}=n \times P\left(C_{n}\right)$. General requirement is $1 \leq \sigma^{g} \leq 2$ and $\sigma^{b}=2-\sigma^{g}$. When $\sigma^{g}=2$ and $\sigma^{b}=0$, existence expected number of the worst individual in next generation is 0 and selection probability of optimal individual is obviously greater than other individuals, which makes premature convergence exist in algorithm. When $\sigma^{g}=\sigma^{b}=1$, selection mode is random selection that is changed to uniform distribution. In general, $\sigma^{g}=1.1$ can be selected.

Calculate individual probability according to formula (3), keep relatively superior individual with roulette wheel selection.

(2) Crossover operation. Adopt single-point crossover way to perform operation. As for two individuals selected, select one crossover point at random, and then perform mutual interchange to priority value at the rear of the point, which is as shown in Figure 2. 


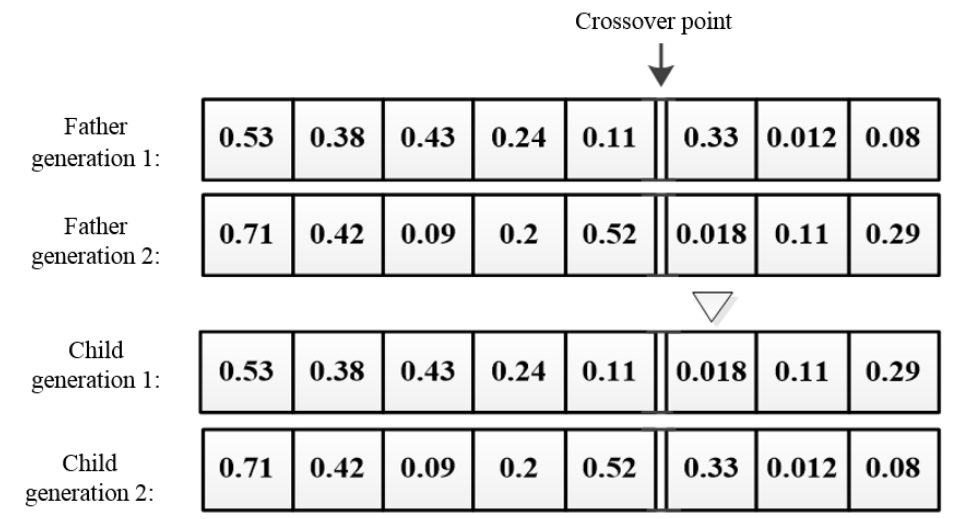

Figure 2. Crossover Operation

(3)Mutation operation. Mutation operator performs operation to individual with mutation probability $\mathrm{Pm}$. Select some priority value of some individual according to mutation probability $P m$, and select value in interval $(0,1)$ at random to replace original value according to uniform distribution.

\section{Server Cluster Load Balancing of ICPSO Algorithm}

Step1: Initialize parameter, such as the maximum iterations $G 1$ and $G 2$, acceleration constant $c 1$ and $c 2$, inertia weight $w$, and population size $N$ etc.

Step2: Calculate particle fitness value of each cluster, and keep location and fitness value of optimal particle. Calculation formula of fitness value is:

$$
f(i)=\frac{1}{\sum X_{i}}
$$

Step3: As for cluster space particle of knowledge space, update location and speed of particle according to formula (5) and formula (6), and perform amplitude limiting processing to their location and speed.

$$
\begin{gathered}
v_{i d}^{t+1}=\omega v_{i d}^{t}+c_{1} r_{1}\left(p_{i d}^{t}-v_{i d}^{t}\right)+c_{2} r_{2}\left(p_{g d}^{t}-x_{i d}^{t}\right) \\
x_{i d}^{t+1}=x_{i d}^{t}+v_{i d}^{t+1}
\end{gathered}
$$

In the formula, $\omega$ is inertia weight; $c 1$ and $c 2$ are learning factor; $r 1$ and $r 2$ are random number within range of $(0,1) ; x_{i}^{t}$ represents location of the $t$ th generation and the ith particle; $v_{i}^{t}$ is velocity vector; $p_{i}^{t}$ represents optimal location of individual; $p_{g}^{t}$ represents global optimal location of population.

Step4: As for each particle, compare fitness function value and extreme value of individual, and if it is greater than optimal value, then replace extreme value of individual with fitness value, and replace extreme value location of individual with location of the particle.

Step5: Compare fitness value of each particle and extreme value of cluster, and if it is more superior to extreme value of cluster, then replace extreme value of cluster with fitness value of the particle, and replace optimal location of cluster with location of the particle.

Step5: Judge if it is true through acceptance function, and if it is true, continuous unimproved algebra of optimal particle fitness of allowable cluster space and knowledge space is exceeded, and then replace particle that has the worst fitness in knowledge space with particle that has optimal fitness in cluster space.

Step7: When iterations exceed the maximum iterations, then search shall be stopped, output optimal location of particle swarm; otherwise, return to Step3 to continue the search. 
Step8: Decode optimal location, and gain optimal scheme of server cluster load balancing.

Working process of ICPSO is as shown in Figure 3.

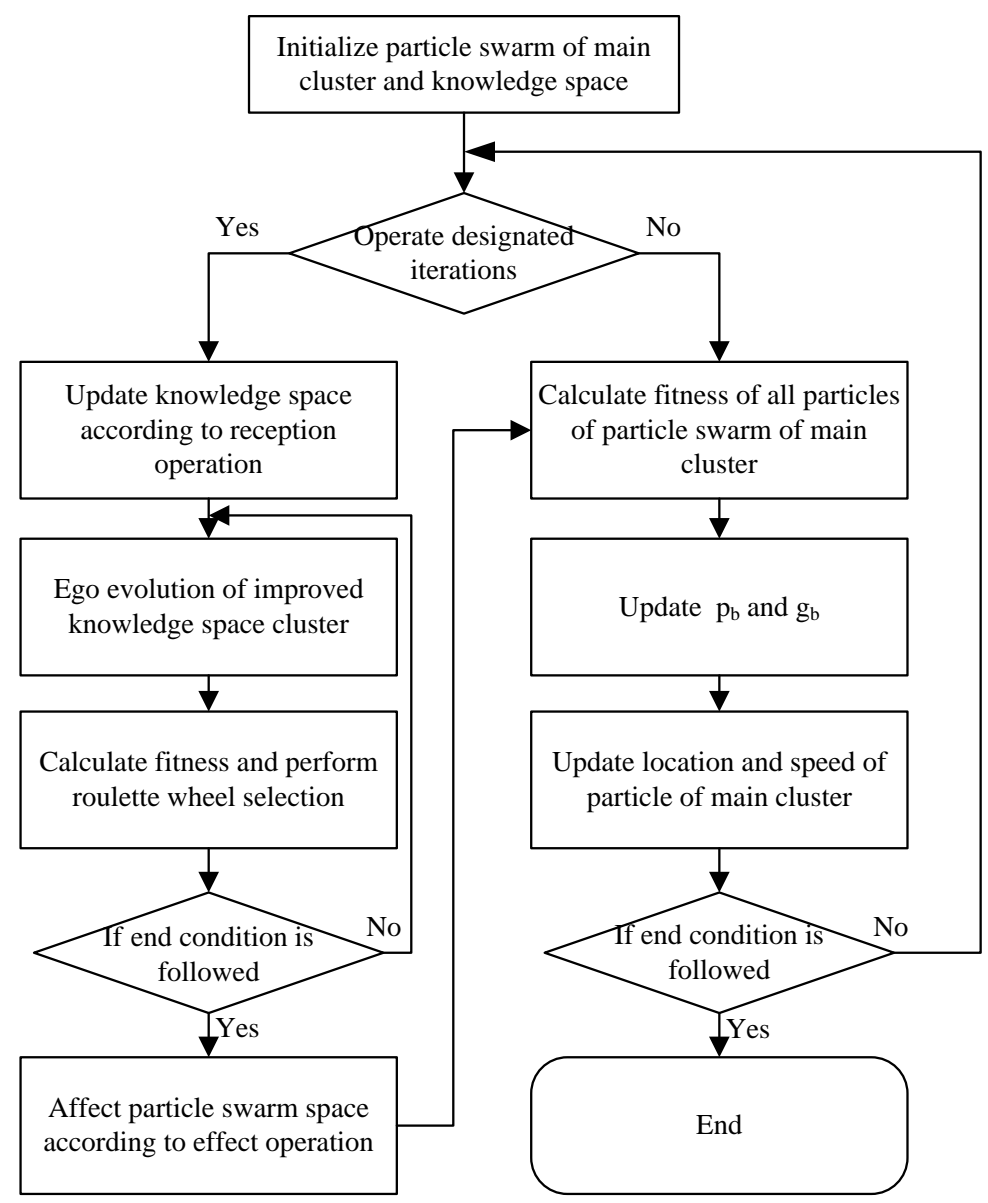

Figure 3. Working Process of ICPSO Algorithm

\section{Simulation Experiment}

\subsection{Simulation Environment}

Server cluster system consists of 1 set of test end, 1 set of management node and 6 sets of servers. Systematic framework is as shown in Figure 4. The entire cluster environment is established in Windows 2000 platform, and Load Rulmer is adopted as simulation testing tool. Performance index of algorithm is: average utilization ratio (\%) of server $\mathrm{CPU}$, average response delay to user request (ms) and average throughput capacity of server cluster system (m). Adopt genetic algorithm (GA) and standard cultural particle swarm optimization algorithm (CPSO) as contrast experiment. Parameter of GA is set as: population size is 20; crossover rate is 0.8 ; mutation rate is 0.02 . Parameter of ICPSO is set as: population size is 50; inertia factor $\omega$ is $1.2 ; C 1$ and $C 2$ are 2; knowledge solution space is $40 \%$ of population size; iterations of all algorithms are 200 times. 


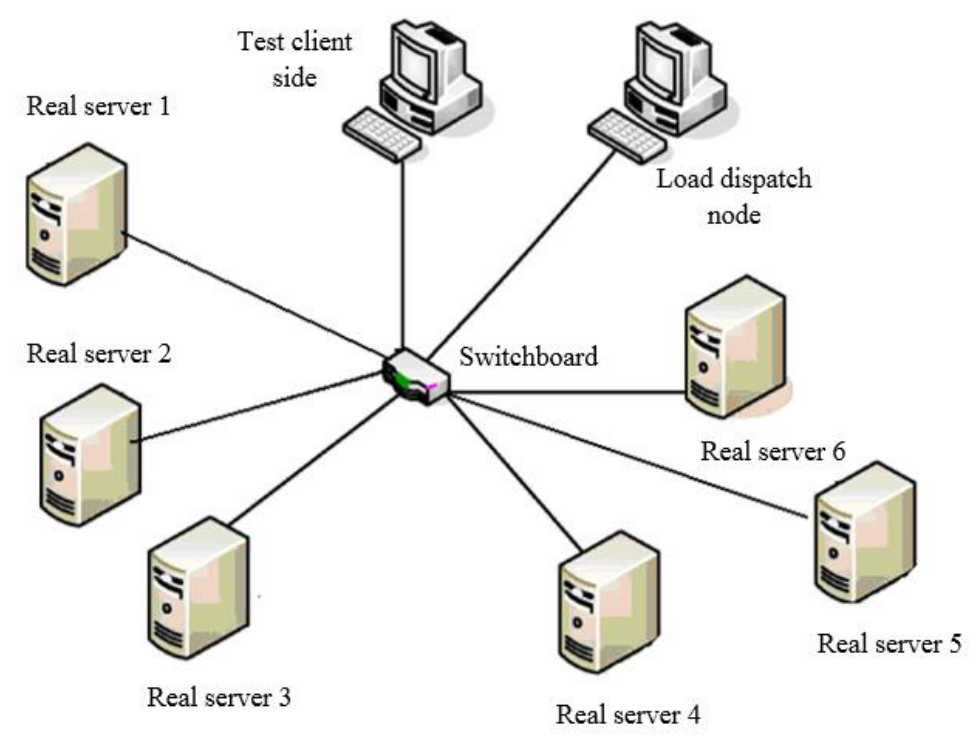

Figure 4. Framework of Server Cluster System

\subsection{Effect of Data Environment}

The chapter mainly researches performance behavior of mentioned algorithm on data of different size and framework by performing simulation experiment to mentioned algorithm on different datasets, thus comprehensively reflecting the entire performance of algorithm. Size of assumed data can be represented as $m \times n$, and 7 groups of testing datasets shall be generated on the basis of IBM data generation equipment, and specific information of these datasets, such as framework and size etc. is as shown in Table 2.

Table 2. Information of Dataset

\begin{tabular}{cccc}
\hline No. & Line & Column & Size \\
\hline$d 2$ & $131072(=217)$ & $64(=26)$ & $64 \mathrm{MB}$ \\
$d 4$ & $262144(=218)$ & $64(=26)$ & $128 \mathrm{MB}$ \\
$d 18$ & $524288(=219)$ & $128(=27)$ & $512 \mathrm{MB}$ \\
$d 19$ & $262144(=218)$ & $256(=28)$ & $512 \mathrm{MB}$ \\
$d 20$ & $131072(=217)$ & $512(=29)$ & $512 \mathrm{MB}$ \\
$d 30$ & $16384(=214)$ & $8192(=213)$ & $1024 \mathrm{MB}$ \\
$d 31$ & $16384(=214)$ & $16384(=214)$ & $2048 \mathrm{MB}$ \\
\hline
\end{tabular}

Table 2 shows 7 groups of testing datasets of different sizes, from 64MB to 2048MB, of which size of dataset $d 2$ is $64 \mathrm{MB}$, and such little dataset can be operated in single computer with little time consumption. But when size of dataset increases to that of dataset $d 30$ and $d 31$, it cannot be operated with single computer. Sizes of 7 groups of testing datasets are unequal, and at the same time, structure (line and column) of data is not totally equal. Speed-up ratio of MapReduce algorithm and iteration time of each time are mainly taken as main indicators in the experiment of this section, and simulation result is as shown in Figure 3 (a) (b).

Definition of evaluation indicator[12]: accelerated speed can be represented as $T_{1} / T_{P}$, and in the formula, ${ }^{T_{1}}$ represents computation time consumed to operate algorithm when there is only one node in MapReduce algorithm; as for subscript of $T_{P}, p=1,2,4,8,16$ represents time consumed to operate MapReduce algorithm when there is $p$ computational nodes. Seen from above definitions of evaluation indicator, accelerated 
speed can reflect resource utilization rate of MapReduce algorithm in a certain degree, and the greater the numerical value of the indicator is, the higher utilization rate of computational resource will be; otherwise, the lower resource utilization rate will be.

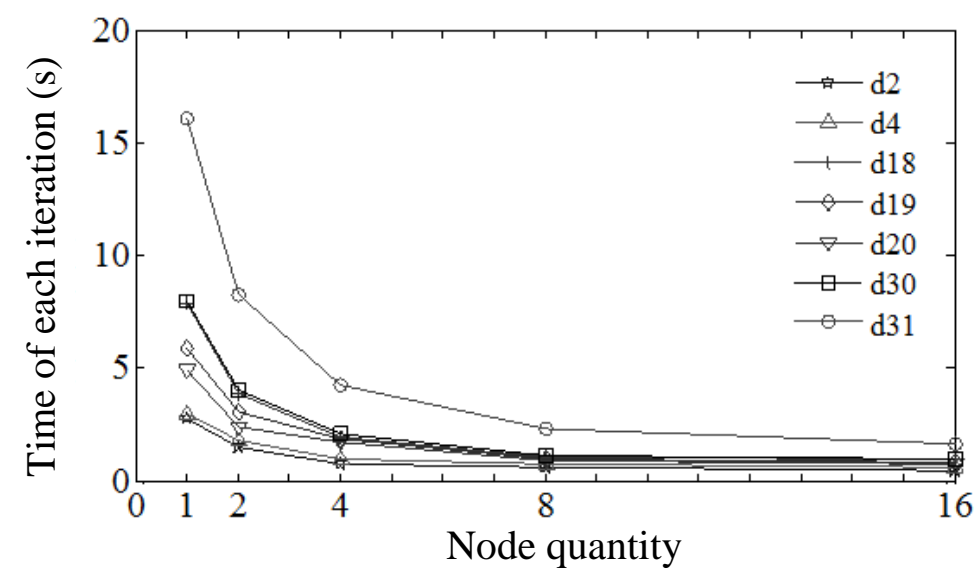

(a) Operation Time and Node Quantity of each Iteration

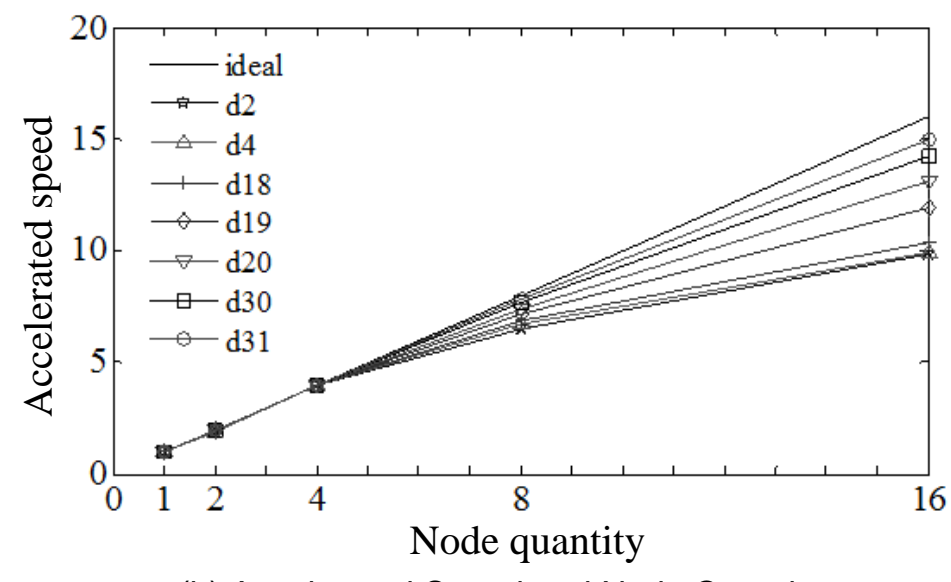

(b) Accelerated Speed and Node Quantity

Figure 5. Comparison of Experimental Results

In Figure 5 (a), abscissa is quantity of node, and ordinate is iteration time at each time, and different curve types represent different datasets. Seen from this figure, under the condition where dataset is constant, iteration time of each step of algorithm decreases with increasing of node quantity, and increasing of computational node quantity is equal to increasing of computational capacity. Operation time will decrease certainly, but because of existence of transmission cost, decreasing tendency is not limitless, and it is not decreased linearly, but tends to balance gradually. Under the condition where node is constant, the greater the dataset is, the longer the operation time will be; the smaller the dataset is, the shorter the operation time will be, and the smaller the node is, the more obvious the difference will be. In Figure 5 (b), abscissa is quantity of node, and ordinate is accelerated speed of algorithm, and different curve types represent different datasets. Seen from this figure., under the condition where dataset is constant, accelerated speed increases with increasing of node quantity. Under the condition where node is constant, the greater the dataset is, the greater the accelerated speed of algorithm operation will be; the smaller the dataset is, the smaller the accelerated speed of algorithm operation will be, and the greater the node quantity is, the more obvious the difference will be. Operation 
advantage of algorithm mentioned in this paper on multiple nodes and big dataset can be shown from the edgewise.

\subsection{Effect of Algorithm Parameter}

Parameter $|h(k)|$ of quantity value of adjacent nodes is involved in above improved algorithm, considering that data range of hypercube structure parameter $|h(k)|$ is:

$$
|h(k)| \in\{2,3, \cdots, 18\}
$$

Seen from Figure 5 (a) (b) in previous section, general variation trend of algorithm on different data is similar. When effect of algorithm parameter $|h(k)|$ on MapReduce algorithm is discussed in this section, above dataset $d 18$ will be selected as representative to perform emulation verification. Hardware equipment condition of experiment is the same with above condition. Referring to previous section, sum of speed-up ratio of algorithm will be adopted as simulation indicator. Simulation result is as shown in Figure 6.

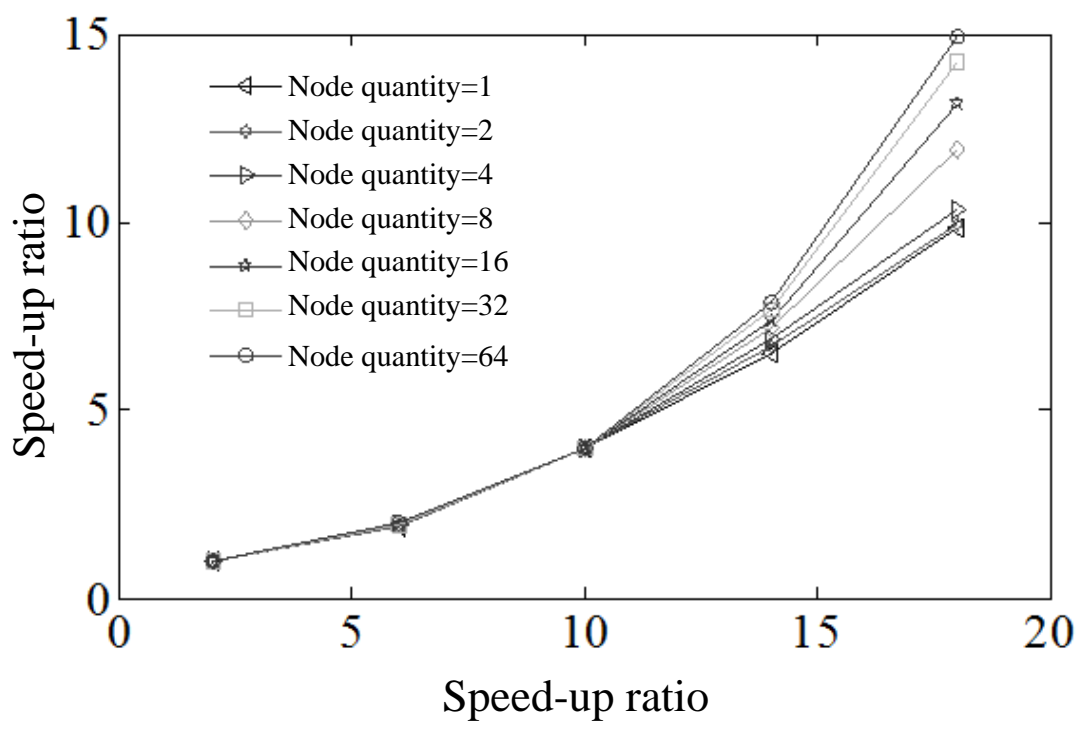

Figure 6. Accelerated Speed and Dataset Parameter

Figure 6 shows speed-up ratio simulation curve of dataset $d 18$ under the condition where value (under general condition, the value is concerned with hypercube structure) of dataset parameter $|h(k)|$ is different and computational resource node quantity is different. Seen from Figure 6, under the condition where computational resource node quantity is constant, speed-up ratio of MapReduce algorithm increases with increasing of dataset parameter. We know that the more elaborate the sampling point selection is, the better the truth of original model will be reflected, but over-elaborate sampling will increase overmuch computational cost. For example, in accelerated speed and dataset parameter selection simulation data shown in Fig.6, speed-up ratio of algorithm increases with increasing of dataset parameter, and operation speed will quicken, but matching rate with original distribution decreases from original $95.6 \%$ to $80.2 \%$. As for dataset $d 18$, we adopt compromise selection method through data simulation, selecting $|h(k)|=9$, and then model matching rate is $91.2 \%$. 


\subsection{Comparison of Algorithm Performance}

After effect of above data environment and algorithm parameter on MapReduce algorithm performance is researched, simulation comparison between algorithm in this paper and comparison algorithm in speed-up ratio performance is mainly verified through simulation. As mentioned earlier, simulation parameter selected in this section is: dataset parameter $|h(k)|=9$, and other computational hardware equipment is the same with above condition. Standard MapReduce, method in literature [13], method in literature [14], method in literature [15] and method in this paper are selected in comparison algorithm, and comparison result is as shown in Figure 7.

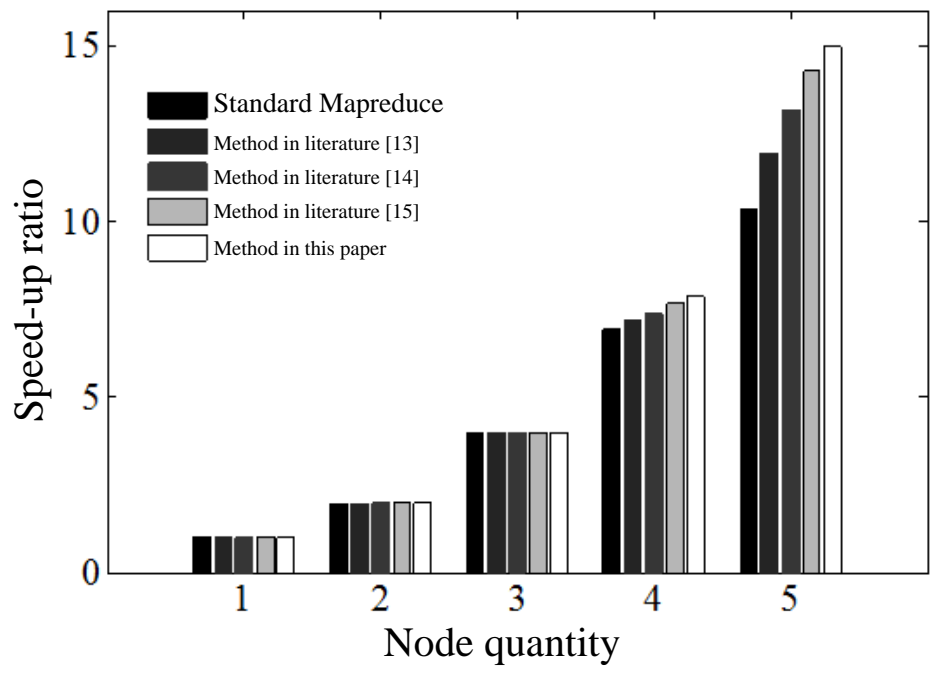

Figure 7. Comparison of Algorithm Performance

Figure 7 shows speed-up ratio comparison simulation figure of 5 kinds of algorithms under different nodes. It can be seen that in several above-mentioned algorithms, when computational resource node quantity is few, for example when node is selected as $1 \sim 3$, difference of speed-up ratio of several comparison algorithms is not obvious, and it is mainly because when computational resource is too few, service efficiency of each computer maximized, and advantage of algorithm in dispatching computational resource cannot be embodied. With increasing of computational resource node quantity, when node quantity is 5 , relative difference of speed-up ratio of several comparison algorithms is obvious. Algorithm performance and speed-up ratio performance of algorithm mentioned in literature [13], literature [14] and literature [15] are different slightly, but they are better than standard MapReduce algorithm, which shows that these improved methods are effective. Speed-up ratio of algorithm mentioned in this paper is obviously better than that of other four comparison algorithms, which shows that when multiple computational resource coordination cooperation handling is required in handling with big data problem, algorithm in this paper can show better coordination computation performance.

\subsection{Result and Analysis}

Adopt GA, CPSO and ICPSO to perform simulation experiment. Gather CPU utilization rate of server in $1 \mathrm{~s}$ time interval. CPU utilization rate of 5 groups of experiment is as shown in Figure 8; average response time of server cluster system dispatch is as shown in Figure 9; throughput capacity of server cluster system is as shown in Figure 10. 


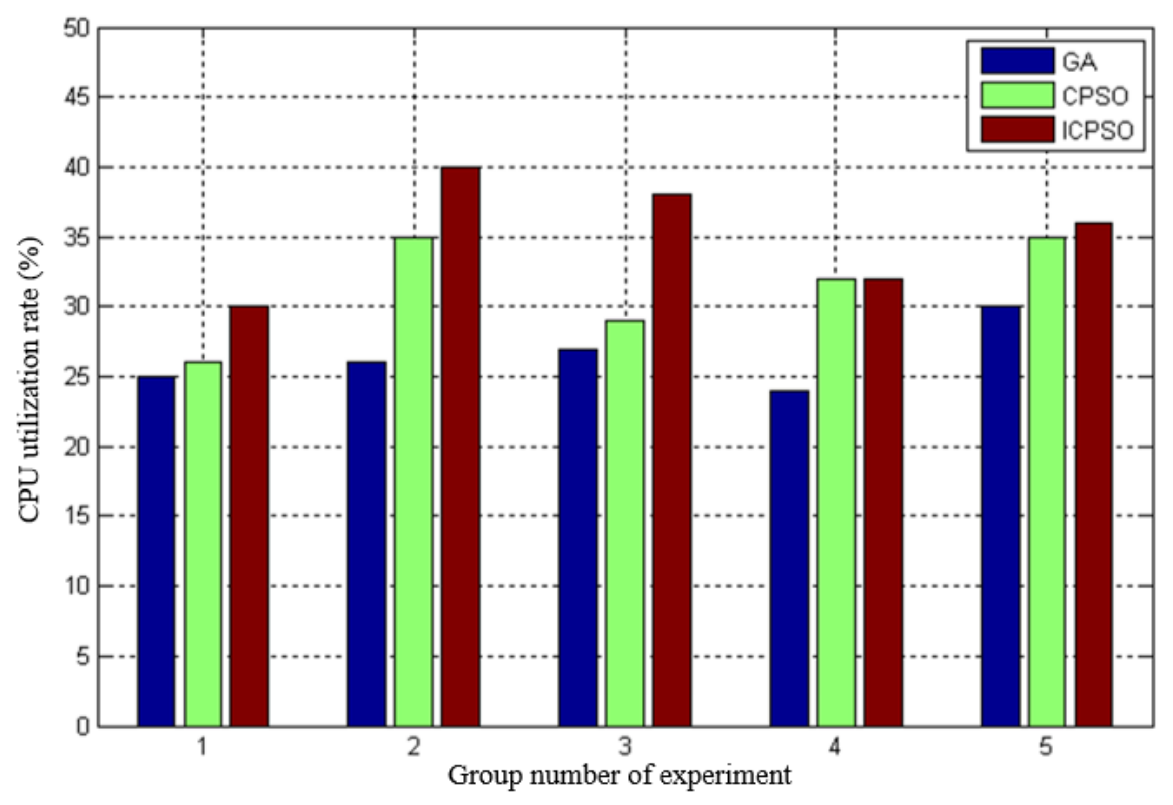

Figure 8. Comparison of CPU Utilization Rate

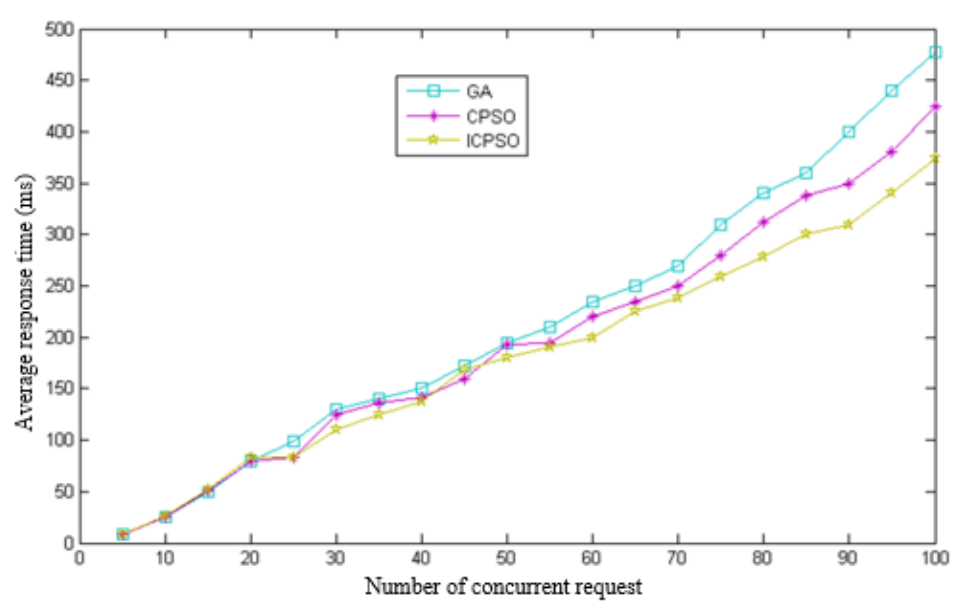

Figure 9. Comparison of Average Response Time

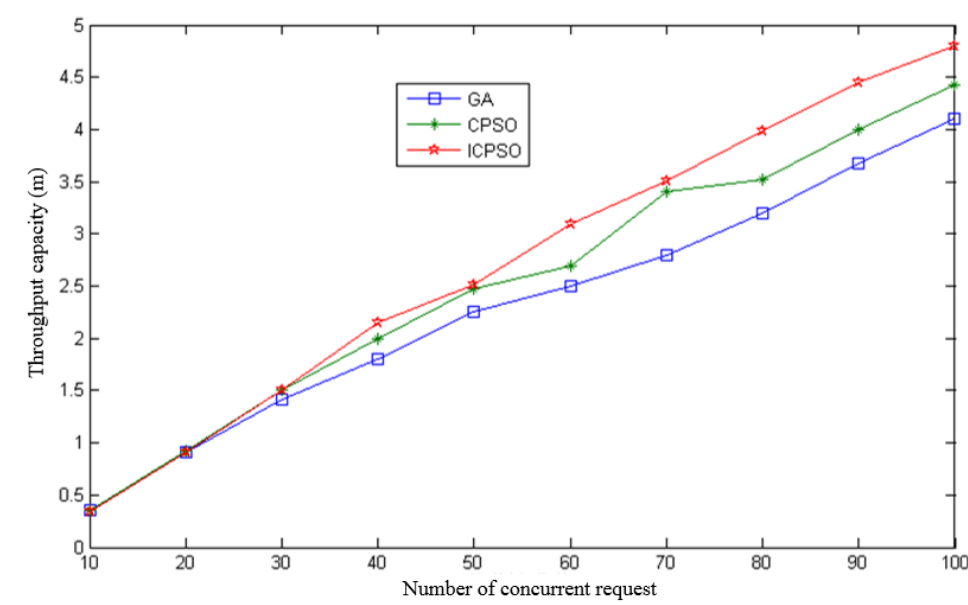

Figure 10. Comparison of Throughput Capacity of System 
Analyze results of Figures 8 10 and we can know that overall performance of ICPSO is superior to algorithm GA and CPSO, and following conclusions can be given:

(1) CPU utilization rate of GA algorithm is the smallest, and it is mainly because local optimum and early mature defect exist in GA algorithm. CPU of CPSO algorithm is greater than GA algorithm, and it is mainly because cultural algorithm and particle swarm optimization algorithm complement each other, and more superior server cluster load dispatch scheme can be gained. CPU utilization rate of ICPSO is the highest. Comparison result shows that mechanism of "double evolution and double promotion" is formed by main cluster space that adopts particle swarm optimization algorithm and knowledge space of cultural algorithm, which improves global searching capacity of algorithm and operational efficiency. Avoid ego evolution restriction of knowledge space of cultural algorithm by bringing in evolution mechanism of GA. Global optimum server cluster load dispatch scheme can be gained, and utilization rate of system resource is improved.

(2) With increasing of number of user request, average response delay time of GA, CPSO and ICPSO increases correspondingly, of which time increasing range of GA is the greatest, and time increasing range of ICPO algorithm is relatively small, and curve change is relatively mild. Comparison result shows that compared with GA and CPSO algorithm, ICPSO algorithm quickens average response speed of user request, and especially for large-scale cluster system, superiority of ICPSO is more obvious.

(3) System throughput capacity of ICPSO algorithm is obviously greater than GA and CPSO algorithm, which shows that server cluster system load scheme gained through ICPSO algorithm is more applicable to large-scale server cluster system load balancing processing.

\section{Conclusions}

Aimed at defect existing in standard particle swarm optimization algorithm, improved cultural particle swarm optimization algorithm is put forward. Simulation result shows that improved cultural particle swarm optimization algorithm improves resource utilization rate of server cluster system, making load more balanced, and being an effective and reliable cluster system load balancing algorithm. At the same time, research regarding to how to embody effect of relevant parameter setting of improved cultural particle swarm optimization algorithm on solution result is problem and direction of further research. Simulation result shows that improved cultural particle swarm optimization algorithm improves resource utilization rate of server cluster system, making load more balanced.

\section{Acknowledgement}

The National Science Foundation of China under Grant No. 61502438.

\section{References}

[1] Z. Lv, T. Yin, H. Song and G. Chen, "Virtual Reality Smart City Based on WebVRGIS", IEEE Internet of Things Journal.

[2] X. Li, Z. Lv, J. Hu, B. Zhang, L. Shi and S. Feng, "Xearth: A 3d gis platform for managing massive city information", In 2015 IEEE International Conference on Computational Intelligence and Virtual Environments for Measurement Systems and Applications (CIVEMSA), IEEE, (2015) June, pp. 1-6.

[3] D. Jiang, Z. Xu and Z. Lv, "A multicast delivery approach with minimum energy consumption for wireless multi-hop networks", Telecommunication systems, (2015), pp. 1-12.

[4] Q. Wang, H. Chen and Y. Shen, "Decision Tree Support Vector Machine based on Genetic Algorithm for fault diagnosis", IEEE International Conference on Automation and Logistics, IEEE, (2008), pp. 2668-2672. 
[5] L. Sun, S. Yoshida and Y. Liang, "A Support Vector and K-Means Based Hybrid Intelligent Data Clustering Algorithm", Ieice Transactions on Information \& Systems, vol. 94-D, no. 11, (2011), pp. 2234-2243.

[6] G. Levitin, J. Rubinovitz and B. Shnits, "A genetic algorithm for robotic assembly line balancing", European Journal of Operational Research, vol. 168, no. 3, (2006), pp. 811-825.

[7] Y. Hacoupian, "Mining Aspects through Cluster Analysis Using Support Vector Machines and Genetic Algorithms", Dissertations \& Theses - Gradworks, (2013).

[8] T. Kanimozhi and K. Latha, "An integrated approach to region based image retrieval using firefly algorithm and support vector machine", Neurocomputing, vol. 151, (2015), pp. 1099-1111.

[9] S. Wu and G. Wei, "High dimensional data Clustering Algorithm Based on Sparse Feature Vector for Categorical Attributes", Logistics Systems and Intelligent Management, 2010 International Conference on. IEEE, (2010), pp. 973-976.

[10] H. Hong, G. Juan and W. Ben, "An Improved KNN Algorithm Based on Adaptive Cluster Distance Bounding for High Dimensional Indexing", Global Congress on Intelligent Systems. IEEE Computer Society, (2012), pp. 213-217.

[11] C. Deng, Y. Liu and L. Xu, "A MapReduce-based parallel K-means, clustering for large-scale CIM data verification", Concurrency \& Computation Practice \& Experience, vol. 28, no. 11, (2015), pp. 30963114.

[12] Q. Shi, C. Li and J. Li, "Study on timely scheduling algorithm for load balance based on Support Vector Machine", IEEE Conference Anthology, (2013), pp. 1-4.

[13] L. Feng and Z. Lv, "Plane surface detection and reconstruction using segment-based tensor voting", Journal of Visual Communication and Image Representation, (2016).

[14] H. Guo, X. Li, W. Wang, Z. Lv, C. Wu and W. Xu, "An event-driven dynamic updating method for 3D geo-databases", Geo-spatial Information Science, (2016), pp. 1-8.

[15] F. Zhao, G. Li, R. Zhang, J. Du, C. Guo, Y. Zhou and Z. Lv, "Swarm-based intelligent optimization approach for layout problem", Multimedia Tools and Applications, (2015), pp. 1-17.

[16] J. L. Fattebert, D. F. Richards and J. N. Glosli, "Dynamic load balancing algorithm for molecular dynamics based on Voronoi cells domain decompositions", Computer Physics Communications, vol. 183, no. 12, (2012), pp. 2608-2615.

[17] T. Kanimozhi and K. Latha, "An integrated approach to region based image retrieval using firefly algorithm and support vector machine", Neurocomputing, vol. 151, (2015), pp. 1099-1111. 